\title{
COMPARATIVE FITNESS OF EXPERIMENTAL POPULATIONS OF DROSOPHILA PSEUDOOBSCURA
}

\author{
MONROE W. STRICKBERGER \\ Department of Biology, St Louis University, St Louis
}

\section{INTRODUCTION}

Received I I.ii. 63

THE present experiment was designed to compare a particular measure of fitness, biomass yield or productivity, for populations of Drosophila pseudoobscura carrying different third chromosome karyotypes. As in the experiment performed by Dobzhansky and Pavlovsky (I96I), this fitness measure has been derived from the techniques of BuzzatiTraverso (1955) and Carson (1958), and measures the productivity of a population in terms of weight and number of offspring produced in half-pint culture bottles. When applied to karyotypes whose relative adaptive values were previously evaluated in polymorphic competitive populations, the procedure used also allows a comparison between different estimates of fitness.

Initially, the question posed by Dobzhansky and Pavlovsky (ibid.) was twofold. First, is a mixed population of two third chromosome arrangements more productive than either of the two arrangements kept separately? Secondly, does the rank in order of fitness between the separate component karyotypes parallel, in terms of biomass productivity, the rank-order of relative adaptive value in a polymorphic population? Dobzhansky and Pavlovsky found that, for the Arrowhead (AR) and Chiricahua $(\mathrm{CH})$ arrangements used, the $A R / C H$ population produced significantly more biomass than AR/AR, but showed no significant difference from $\mathrm{CH} / \mathrm{CH}$. Furthermore, the order of fitness for biomass productivity between the $A R / A R$ and $\mathrm{CH} / \mathrm{CH}$ karyotypes was markedly changed in comparison to the relative adaptive values previously established for these arrangements in population cage experiments (Dobzhansky and Pavlovsky, 1960).

However, since the biomass experiments of Dobzhansky and Pavlovsky concerned populations which had long been kept isolated from each other in polymorphic (AR/CH) and monomorphic $(\mathrm{AR} / \mathrm{AR}$, $\mathrm{CH} / \mathrm{CH}$ ) laboratory cages, the problem of comparing karyotypes originating from the same polymorphic population remained to be undertaken. The significance of this distinction lies in the fact that the relative adaptive value of an arrangement can be considerably modified by its past selective environment, and a particular karyotype (i.e. $\mathrm{CH} / \mathrm{CH}$ ) derived from a monomorphic cage and from a polymorphic cage are not comparable in fitness (Strickberger, I963). Thus, the biomass productivity tested by Dobzhansky and Pavlovsky for karyotypes long kept in separate population cages need not reflect the relative fitness of the karyotypes derived from a combined population. 
Of the four karyotypic populations tested for biomass production in the experiments presently to be described, three (AR/CH I73, AR/AR $173, \mathrm{CH} / \mathrm{CH}{ }_{1} 73$ ) were derived from a common polymorphic laboratory cage, \#I 73 . In cage $I 73$ the relative values of the three karyotypes had been previously determined (Dobzhansky and Pavlovsky, 1960) and both the AR and CH arrangements had the opportunity to become " coadapted" to each other over a period of more than three years. On the other hand, the fourth population tested, $\mathrm{CH} / \mathrm{CH} \mathrm{I}_{77}$, was derived from cage $\mathrm{I} 77$ which had been maintained the same length of time but in monomorphic condition, i.e., without the presence of AR. In previous experiments (Strickberger, ibid.) the competitive fitness of the $\mathrm{CH}$ gene arrangement as compared to a standard AR arrangement had been determined for both $\mathrm{CH}$ isolated from cage $\mathrm{I} 73$ and from cage $\mathrm{I} 77$. In addition, both the fitness of entire populations from polymorphic cage 173 and from monomorphic cage 177 had been tested in interspecific competition with a mutant strain of $D$. melanogaster.

Three problems of comparative fitness were therefore approached in this experiment. First, does biomass productivity, measured according to the method to be described, reflect the relative adaptive values of the different karyotypes within the same population? Second, does biomass productivity reflect the competitive ability of the same karyotypes from different populations? Third, does biomass productivity reflect the fitness under interspecific competition of two different D. pseudoobscura populations? Also, once the present experiment was well on its way, an attempt was made to discover whether the periodic transfer of a few flies from one of the test populations to another would result in the heterosis observed by Carson (1958) when transferring a single haploid set of chromosomes from one $D$. melanogaster population to another.

In evaluating the results of the present experiment, and in an attempt to understand fitness measurements in general, advantage has been taken of the considerable further information which has accumulated in the literature for the third chromosome arrangements studied (Dobzhansky, 1947; Beardmore et al., 1960). All pertinent fitness estimates have, therefore, been tabulated and comparisons made between them.

\section{MATERIALS AND METHODS}

Population cages 173 and 177 , furnishing the material for the present experiments, were established in 1957 from strains which originated ro or more years previously in Pinon Flats, San Jacinto Mountains, California (Dobzhansky and Pavlovsky, 1960). Population 173 was founded with ro or more strains of each third chromosome type, at 20 per cent. $\mathrm{AR}$ and 80 per cent. $\mathrm{CH}$, and reached an cquilibrium frequency in 20 months' time of about 70 per cent. AR and 30 per cent. $\mathrm{CH}$. Population 177 , with the same starting date, consisted wholly of a mixture of all the various strains homozygous for $\mathrm{CH}$. In the summer of 1960 , the author isolated 13 or more strains each of AR and $\mathrm{CH}$ karyotypes from the 173 population. 
The isolation technique made each strain isogenic for the third chromosome arrangement with 96 per cent. or more of the remainder of the genetic background from the same 173 population (Strickberger, 1963). These strains were maintained individually until January 1961 , and then crossed with each other to furnish founding populations of 75 pairs of flies for each of the I 73 populations that were to be tested for biomass productivity: $\mathrm{AR} / \mathrm{CH}$ I $_{73}, \mathrm{AR} / \mathrm{AR}$ I $73_{3}, \mathrm{CH} / \mathrm{CH}_{173}$. At the same time as these test populations were founded, a similar number of adult flies were randomly removed from the $\mathrm{CH}$ monomorphic cage $\mathrm{I} 77$ to begin the $\mathrm{CH} / \mathrm{CH}$ test population. Each test population was replicated with the designation of either $A$ or $B$ (i.e., 8 populations in toto) and kept at $25^{\circ} \mathrm{C}$. in half-pint cultures on a Cream-of-wheatmolasses medium. Each culture was moistened with one drop of a freshly made suspension of Fleischmann's Dry Yeast (one gram per 1o c.c. distilled water) and a uniform strip of sterilised paper towelling was inserted in the food.

TABLE I

\section{Data on test populations}

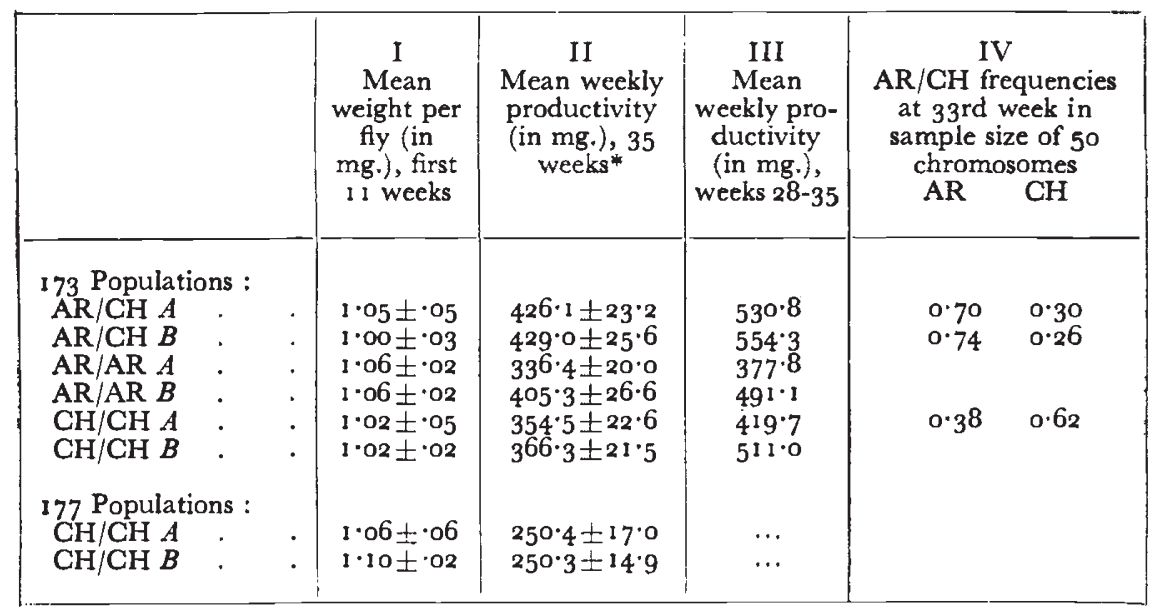

* 33 weeks for 177 populations.

Twice weekly (Mondays and Thursdays) transfers were made to newly prepared culture bottles for each test population. On one of these weekly occasions, the flies that had emerged from previous cultures were etherised, counted (for the first II weeks), and weighed. At that time, 75 pairs of these newly emerged flies (first 11 weeks) or a weighed quantity of approximately 150 such flies ( 155 milligrams) were used as the new adult population for each test group, discarding meanwhile the old adult population that had been used as parents for the previous week. In this fashion, the parental size of each test population remained uniformly at about 150 one-iveek-old flies throughout the experiment. (In the first two or three weeks of the experiment, before new flies emerged, the parental group for each test population were counted, and flies were added as needed from the cultures that had initially furnished the founding population.) As compared to previous experiments of this type in which both old and newly emerged flies are transferred en masse to new cultures, the present technique of restricting parental population size to 1 jo adults considerably diminished adult crowding and egg trampling, and eliminated large differences in parental age. Also eliminated was the initial "explosion " of the population sizes to over rooo flies for the first few months with a consequent " crash" as reported by Dobzhansky and Pavlovsky (1961). 
As stated above, for the first II weeks of the experiment, the newly emerging flies from each test population were both counted and weighed. This gave an average weight per fly with standard error as shown in column I of table I. Since there did not appear to be any evident departure from a $1:$ i sex ratio in any of the populations, it was decided that counting could be eliminated and a random sampling of fies weighing $155^{\circ} 0$ milligrams would furnish approximately 150 adults evenly divided between the sexes. Therefore, from the 12 th week on, this weighed quantity of flies was separated from the weighed amount of newly emerged flies in each test group and used as the parental population until the following week. In test populations of $\mathrm{CH} / \mathrm{CH} \mathrm{I}_{77}$, flies that had been used as parents the previous week were added to furnish the desired amount in those few instances when 150 newly emerged flies had not been produced for collection. The weighings were made on a chemical balance sensitive to o. I milligrams which was used by Dobzhansky and Pavlovsky.

After the populations had been continued for 26 weeks, 5 pairs of newly emerged flies from the AR/AR $A \mathrm{I} 73$ population were transferred weekly to the $\mathrm{CH} / \mathrm{CH}$ $A$ I 73 population until the 3 ist week. At the 33 rd week of the experiment egg samples were taken from both of the AR/CH 173 populations and also from the $\mathrm{CH} / \mathrm{CH} A$ 173 populations which had been receiving $A R / A R$ flies. The larvæ from these eggs were raised under optimum conditions, at $19^{\circ} \mathrm{C}$. in well-yeasted cultures, and $5^{\circ}$ salivary chromosomes examined from each of the three populations.

The experiment was terminated when it had proceeded for 35 weeks for the 173 test populations and for 33 weeks for the $\mathrm{I} 77$ populations.

\section{RESULTS}

The means and standard errors (in milligrams) for the weekly weighings of flies produced by each population for the entire experiment are reported in column II of table $\mathrm{I}$. A table of the individual weekly weights for each population is appended. * Despite the rather high standard errors, there are notable differences between the means of the 173 populations derived from the polymorphic cage and those of the $\mathrm{I} 77$ populations derived from the $\mathrm{CH}$ monomorphic cage. This difference is significant at the 0.05 level in every comparison and at even lower levels in most cases. Among the karyotypes within the $\mathrm{I} 73$ group, the biomass productivity of the $\mathrm{AR} / \mathrm{CH}$ populations appears superior to the homozygous component populations, although the differences, for the most part, are not clearly statistically significant. Comparisons of means between AR/AR I 73 and $\mathrm{CH} / \mathrm{CH}$ I 73 show no clear superiority of either group, although the average of the AR/AR's is somewhat higher than the average of $\mathrm{CH} / \mathrm{CH}$ 's.

The effect on biomass productivity of the weekly transfer of five pairs of AR/AR 173 flies into the $\mathrm{CH} / \mathrm{CH}$ I 73 population is reported in column III of table I. This column shows the mean productivity for each of the 173 populations starting from the 28 th week, two weeks after the introduction of AR/AR flies was initiated, until the end of the experiment, eight weeks later. Although there is a relative rise in mean biomass production during this period for all the 173 populations, the rise for the $\mathrm{CH} / \mathrm{CH} A$ I 73 populations (the recipient of the AR/AR flies) is clearly less than the rise in mean for its replicate, $\mathrm{CH} / \mathrm{CH} B \mathrm{I} 73$.

Column IV of table I reports the frequencies of the $\mathrm{AR}$ and $\mathrm{CH}$ arrangements in 50 chromosomes in the $33^{\text {rd }}$ week from egg samples 
of the I 73 populations, $\mathrm{AR} / \mathrm{CH} A, \mathrm{AR} / \mathrm{CH} B$, and $\mathrm{CH} / \mathrm{CH} A$ (recipient of $\mathrm{AR} / \mathrm{AR} A$ ). In the two $\mathrm{AR} / \mathrm{CH}$ populations the frequencies closely approximate the equilibrium frequencies of these same arrangements in the population cage itself (Dobzansky and Pavlovsky, I96o; Druger, unpublished). In the $\mathrm{CH} / \mathrm{CH} A$ population, AR had become firmly established within six weeks after its original introduction.

\section{COMPARISON OF FITNESS ESTIMATES}

Various fitness estimates of the particular arrangements tested in this experiment have been given for a variety of conditions. These data

TABLE 2

Fitness data for designated Arrowhead and Chiricahua karyotypes and populations

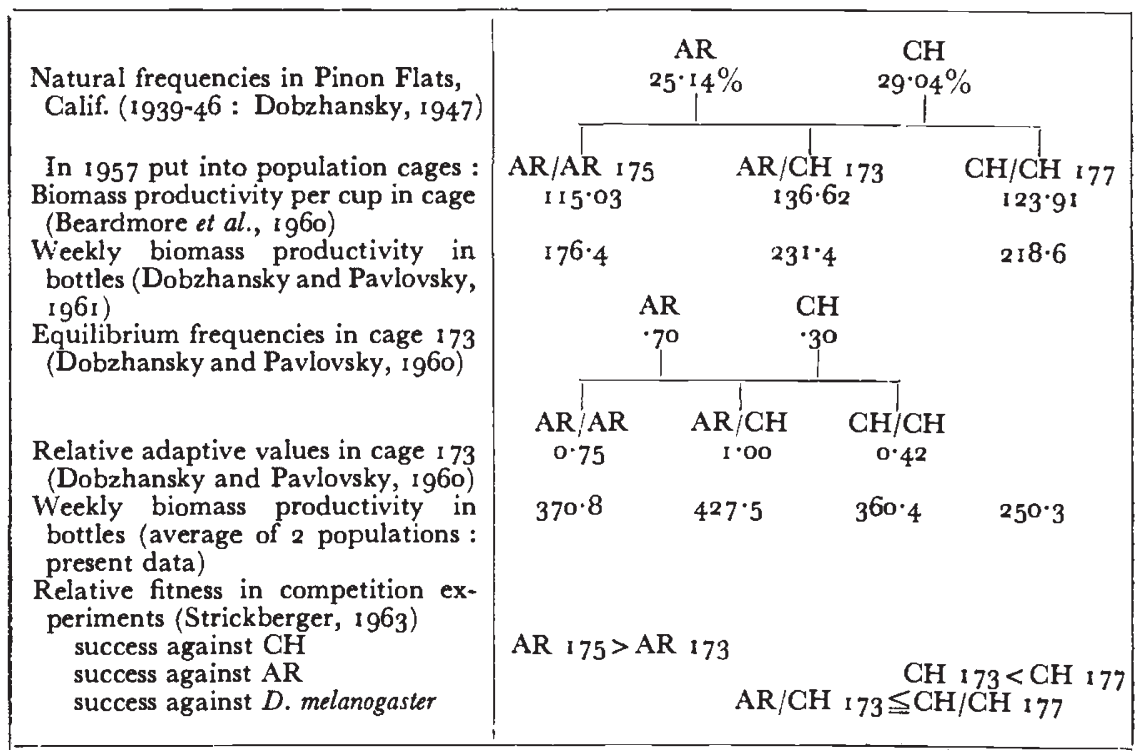

have been tabulated (table 2), and the following comparisons can bc made:

I. The relationship between the laboratory equilibrium frequencies of a polymorphic population of $\mathrm{AR} / \mathrm{CH}$ (cage I 73: Dobzhansky and Pavlovsky, I 960) does not reflect the frequencies of these arrangements under the natural conditions in which other arrangements are also present (Dobzhansky, I947).

2. Biomass productivity in either population cage cups (Beardmore et al., 1960) or bottles (Dobzhansky and Pavlovsky, I96I) does not reflect the relative adaptive values of the karyotypes in the polymorphic cage (Dobzhansky and Pavlovsky, 1960). (In fact, for the first 18 weeks of data discarded by Dobzhansky and Pavlovsky (196I) because of adult crowding, the usual relative adaptive values in the polymorphic cage were exactly reversed, $\mathrm{CH} / \mathrm{CH}>\mathrm{AR} / \mathrm{AR}>\mathrm{AR} / \mathrm{CH}$.) 
3. Biomass productivity of karyotypes isolated from the same polymorphic cage (present data) is also not a clear reflection of relative adaptive values in the cage.

4. The competitive success of a karyotype when tested both intraand interspecifically $(\mathrm{CH} / \mathrm{CH}$ 177: Strickberger, 1963$)$ is by no

TABLE 3

Weekly productivity (in milligrams)

\begin{tabular}{|c|c|c|c|c|c|c|c|c|}
\hline \multirow{3}{*}{ Weeks } & \multicolumn{6}{|c|}{ I 73 Populations } & \multirow{2}{*}{\multicolumn{2}{|c|}{$\frac{177 \text { Populations }}{\mathrm{CH} / \mathrm{CH}}$}} \\
\hline & \multicolumn{2}{|c|}{$\mathrm{AR} / \mathrm{CH}$} & \multicolumn{2}{|c|}{$\mathrm{AR} / \mathrm{AR}$} & \multicolumn{2}{|c|}{$\mathrm{CH} / \mathrm{CH}$} & & \\
\hline & $A$ & $B$ & $A$ & $B$ & $A$ & $B$ & $A$ & $B$ \\
\hline $0-2$ & $\ldots$ & & & & & & $\ldots$ & $\ldots$ \\
\hline 3 & $100 \cdot 3$ & $225^{\prime 2}$ & $121 \cdot 2$ & 221.5 & $\log 0$ & $230 \times 2$ & 77.9 & 114.2 \\
\hline 4 & $457^{\circ}$ & $435 \cdot 5$ & 162.4 & 350.2 & 148.3 & 2154 & 176.5 & $183^{7} 1$ \\
\hline $\begin{array}{l}7 \\
5\end{array}$ & 3214 & 319.1 & 360.4 & 268.9 & $\begin{array}{r}178.6 \\
\end{array}$ & 488.8 & 65.5 & 106.3 \\
\hline 6 & 3145 & $5^{16.1}$ & $217 \cdot 6$ & 383.0 & $257 \cdot 1$ & 407.0 & 94.7 & 270.0 \\
\hline 7 & 268.0 & $5^{27} \cdot 3$ & 267.9 & 238.4 & $201 \cdot 6$ & 317.9 & $247 \cdot 3$ & $221 \cdot 9$ \\
\hline 8 & 353.4 & 322.9 & 180.8 & $579 \cdot 9$ & $230 \cdot 9$ & 230.4 & 296.7 & 341.8 \\
\hline 9 & 369.1 & 306.4 & $278 \cdot 1$ & $\begin{array}{l}2922 \\
\end{array}$ & 393.5 & $323 \cdot 4$ & 326.5 & 187.6 \\
\hline 10 & 306.9 & $455^{\prime 2}$ & $405^{\prime 2}$ & $5^{28} \cdot 4$ & 240.0 & 439.4 & $396 \cdot \mathrm{I}$ & 253.5 \\
\hline II & 610.6 & $55^{1} \cdot 4$ & 286.4 & 654.5 & 3654 & $398 \cdot 7$ & 213.4 & $317 \cdot 1$ \\
\hline 12 & $356 \cdot 8$ & 253.6 & 2457 & 179.6 & 421.6 & $259 \cdot 7$ & 2447 & $304 \cdot 5$ \\
\hline 13 & 376.5 & $33^{2} \cdot 2$ & 198.6 & 286.8 & 267.8 & 220.0 & 331.5 & $230 \cdot 3$ \\
\hline 14 & 283.9 & $3^{66.9}$ & 2454 & 366.7 & 383.8 & $409 \cdot 9$ & $242 \cdot 3$ & $304 \cdot 3$ \\
\hline $\begin{array}{l}7 \\
15\end{array}$ & $590^{\circ} \cdot 2$ & 353.7 & 339.6 & 4543 & $444 \cdot 8$ & $252^{2 \cdot 2}$ & $349 \cdot 2$ & $15^{1} .0$ \\
\hline 16 & $492 \cdot 1$ & $330 \cdot 3$ & $34^{8 \cdot 1}$ & $369 \cdot 3$ & 267.5 & $319 \cdot 2$ & $229^{\prime}$ & 165.4 \\
\hline 17 & 412.3 & $35^{6 \cdot 5}$ & $543 \cdot 1$ & $532 \cdot 3$ & 460.8 & $190^{\circ} 4$ & $171^{\circ} 0$ & $233 \cdot 1$ \\
\hline 18 & 315.4 & 296.2 & $333 \cdot 6$ & 384.2 & $319^{\circ}$ & 188.3 & $279^{\circ}$ & 398.3 \\
\hline 19 & $295^{\circ}$ & $394^{\prime 2}$ & $259^{\prime 2}$ & 325.4 & $268 \cdot 5$ & $252 \cdot 8$ & $246 \cdot 2$ & $278^{\circ} 2$ \\
\hline 20 & $478 \cdot 2$ & $43^{\circ} \cdot \mathrm{I}$ & 372.4 & 482.5 & 474.8 & $409 \cdot 2$ & $438 \cdot I$ & $372 \cdot 8$ \\
\hline 21 & $279^{\circ} 5$ & 307.8 & $220^{7}$ & 171.7 & 238.4 & $346 \cdot 6$ & 275.5 & \\
\hline 22 & $54^{\circ} \cdot 3$ & $443^{\circ} \circ$ & 3944 & 6104 & 393.8 & 403.4 & 270.6 & 288.8 \\
\hline 23 & 545.9 & 410.2 & $54: 4$ & $34^{6 \cdot 2}$ & $659^{\circ} 6$ & $315^{\circ}$ & 173.3 & 134.8 \\
\hline 24 & $4^{26} \circ$ & $433^{\prime} \cdot$ & $5^{26.0}$ & 377.8 & $54^{2} \cdot 5$ & $359^{\circ} 4$ & 326.6 & $184^{\prime}$ \\
\hline 25 & 417.8 & $3^{2} 4 \cdot 6$ & 522.7 & $349^{\circ} 7$ & 417.6 & 355.7 & $15^{1} \cdot 9$ & $176 \cdot 3$ \\
\hline 26 & $579^{\circ} \mathrm{I}$ & 4151 & $387 \cdot 3$ & 323.4 & 277.3 & 342.4 & 223.4 & 274.5 \\
\hline 27 & $3^{26 \cdot 6}$ & 613.2 & 323.2 & $362 \cdot 0$ & $378 \cdot 1$ & $324 \cdot 1$ & $223^{\circ}$ & $23^{2} \cdot 2$ \\
\hline 28 & $4177^{\circ}$ & 5954 & $\begin{array}{r}46.9 \\
463 .\end{array}$ & $500^{\circ}$ & $397 \cdot 6$ & 357.6 & $478 \cdot 2$ & 235.1 \\
\hline 29 & $423 \cdot 2$ & 667.3 & 342.4 & 493.4 & 376.3 & $462 \cdot 0$ & 258.9 & $44^{6 \cdot 8}$ \\
\hline 30 & 729.5 & 446.7 & 277.8 & $45^{6 \cdot 7}$ & $55^{6 \cdot 6}$ & $489 \cdot 7$ & $26 \mathrm{~K}^{\prime} \cdot 4$ & 344.7 \\
\hline 31 & 584.7 & 607.3 & .506 .4 & $539^{\circ} 7$ & $477 \cdot 1$ & $563^{\circ} \circ$ & $24^{1} \cdot 2$ & $\begin{array}{l}172.3 \\
\end{array}$ \\
\hline 32 & 641.7 & 854.3 & $330^{\circ} 3$ & 918.1 & $310 \cdot 2$ & 768.0 & 157.4 & 252.8 \\
\hline 33 & 5143 & 194.6 & Oי & 267.0 & $608 \cdot 7$ & $472 \cdot 8$ & $294^{\circ}$ & $275^{\prime}$ \\
\hline 34 & $4^{16} \cdot 3$ & $708 \cdot 7$ & $329^{\prime 2}$ & $455^{\circ} 0$ & $3^{18 \cdot 5}$ & $504 \cdot 2$ & $\ldots$ & \\
\hline 35 & $5^{19} \cdot 3$ & $3^{60} \cdot 0$ & $281 \cdot 1$ & 298.7 & $312 \cdot 8$ & 470.7 & 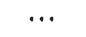 & $\cdots$ \\
\hline
\end{tabular}

means a measure of its relative productivity when tested in bottles (present data).

5. Different methods for testing biomass productivity in bottles (Dobzhansky and Pavlovsky, 196I; present data) give different relative productivities for the same karyotypes $(\mathrm{AR} / \mathrm{CH}$ 173, $\mathrm{CH} / \mathrm{CH}$ I 77). 


\section{DISCUSSION}

The present experiments are concerned with estimating the relative fitness of individual populations of Drosophila pseudoobscura monomorphic for Arrowhead (AR/AR), or for Chiricahua $(\mathrm{CH} / \mathrm{CH})$, or polymorphic for both of these third chromosome arrangements. The fitness measurement has been biomass productivity, the weekly weight of adults produced by each population isolated in culture bottles. As used by Dobzhansky and Pavlovsky (196I), this technique showed a significantly higher biomass productivity of the $\mathrm{AR} / \mathrm{CH}$ and $\mathrm{CH} / \mathrm{CH}$ populations in comparison to AR/AR populations, but no significant difference between the two former groups. The experiments reported here have given, in some respects, a quite different order of fitness, in addition to a distinctive difference between populations of the same karyotype $(\mathrm{CH} / \mathrm{CH})$ derived from different experimental cages.

As shown in table 2, the average biomass productivities of populations of karyotypes derived from the same polymorphic cage (I 73) follow, to some extent, the relative fitness values that had previously been established within the cage itself (Dobzhansky and Pavlovsky, 1960). That is, with the exception of $\mathrm{AR} / \mathrm{AR} A$, the order of fitness is $\mathrm{AR} / \mathrm{CH}>\mathrm{AR} / \mathrm{AR}>\mathrm{CH} / \mathrm{CH}$. Also, the selective history of a karyotype was shown significantly to modify its relative productivity, as seen in the lower values of $\mathrm{CH} / \mathrm{CH}$ from monomorphic cage $\mathrm{I} 77$ in comparison to any of the karyotypes from polymorphic cage 173 . The uniqueness of the present findings must, therefore, be discussed within the context of at least two innovations in the plan of the experiment.

The present experiment was able to distinguish between populations of karyotypes isolated from polymorphic or monomorphic sources. Since the competitive fitness of an arrangement was shown to change with mononorphic or polymorphic population cage experience (Strickberger, 1963 ), a difference in biomass productivity for a karyotype from both sources could reasonably be expected. A second innovation was to maintain the parental size of each test population at I 50 one-week-old adults, thereby eliminating variables in respect to population size and crowding.

That these differences in design between the present experiment and that of Dobzhansky and Pavlovsky (I96I) have led to unique results, is therefore not surprising. What is of special interest, however, is the apparent reversal of the fitness order in the present tests as compared to that in competition experiments. Specifically, it had previously been found (Strickberger, 1963) that the most fit population cage competitor when tested against a standard stock of AR was $\mathrm{CH}$ derived from the monomorphic population cage $\mathrm{I} 77$, while $\mathrm{CH}$ from the polymorphic cage I 73 was markedly less fit. Furthermore, when testing an entire $D$. pseudoobscura population against a standard stock of $D$. melanogaster, the monomorphic $\mathrm{CH} / \mathrm{CH}$ population was as good as or better than the $\mathrm{AR} / \mathrm{CH}$ I 73 population. 
Thus, the order of competitive success in population cages is not accompanied by a comparable order of biomass productivity, at least, when tested in bottles. These experiments, therefore, offer support to Mather's contention ( $196 \mathrm{I}$ ) that "selection arising from competition will favour nothing but the ability to survive that particular type of competition. Indeed, the relative rise of a special type of competitive power may be accompanied by a reduction in other components of fitness." The phenomenon of a negative correlation between competitive ability and other fitness components has also been known as the "Montgomery effect" (Montgomery, 1912), and is supported by various findings (Dobzhansky, 1950; Gustafsson, 1951; Sakai and Gotoh, 1955; but see also Lerner and Ho, 1961).

On the other hand, for the karyotypes within the single polymorphic population 173 , biomass production for each karyotype seems to reflect more closely their competitive relations within the cage. In this case, however, some ambiguity still remains since a test population of $\mathrm{AR} / \mathrm{CH}$ flies is continually segregating, in turn, for three karyotypes, $A R / C H$, $\mathrm{AR} / \mathrm{AR}, \mathrm{CH} / \mathrm{CH}$. The superior biomass productivity of such a population may, therefore, arise from interaction between the different karyotypes, as well as from the superior productivity of the heterokaryotypes. Nevertheless, it does seem reasonable to conclude that although there may be no correlation between the relative competitive abilities of populations or karyotypes in one situation and their fitness components in a different situation, such correlation appears more probable for some of the karyotypes derived from within a long-standing polymorphic population. In this respect, it is of interest that the frequencies of the $\mathrm{AR}$ and $\mathrm{CH}$ arrangement in the two $\mathrm{AR} / \mathrm{CH} I 73$ test populations (table I, column IV) approximate, by the 33 rd week, the frequencies in the 173 population cage (Dobzhansky and Pavlovsky, 1960). Evidently, the conditions of competition or facilitation between these particular " coadapted" laboratory arrangements are not radically altered even when combined under somewhat different laboratory conditions.

The much lower frequency of AR chromosomes ( 38 per cent.) in the $\mathrm{CH} / \mathrm{CH} A$ population, recipient of $\mathrm{AR} / \mathrm{AR}$ flies for six weeks, may be a result of the rather short period of time (seven weeks) that had elapsed from the introduction of AR chromosomes to the time of scoring. It is, however, interesting to note that, for the latter part of the experiment (table I, column III), there was no relative increase in population size of $\mathrm{CH} / \mathrm{CH} A$ compared to $\mathrm{CH} / \mathrm{CH} B$, despite the addition of AR chromosomes to the former. These findings differ from the changes reported by Carson $\left(195^{8}, 1961 a\right)$ for the addition of foreign chromosomes to $D$. melanogaster populations, but are similar to Carson's more recent findings in $D$. robusta (Carson, $196 \mathrm{I} b$ ). The difference in species behaviour when new genetic material is being added has been explained by Garson on the assumption that the $D$. robusta populations cannot be further improved by additional polymorphism because of 
their already "saturated" (presumably heterozygous) condition. In the present case, since the AR/CH populations do appear to be more productive than the component homokaryotype populations, the absence of improvement on mixing the component populations seems difficult to explain on the basis of an already "saturated" $\mathrm{CH} / \mathrm{CH}$ population. However, if account is taken of the changes that probably occurred within the component populations during the twenty-six weeks they were carried prior to their "mixing", an explanation may be offered. During this period of time, the many strains of $\mathrm{CH}$ and $\mathrm{AR}$ in the separate $\mathrm{CH}$ and $\mathrm{AR}$ populations could freely recombine and evolve so that "coadaptation" between these two arrangements, or their "mutual compatibility", was no longer the same as when they were initially extracted from the polymorphic population. The finding that a measurable change in relative fitness can occur when a number of $\mathrm{GH}$ (or AR) strains recombine under selective conditions in a laboratory population cage (Strickberger, 1963) supports the view that "coadaptation" between two arrangements can be modified by individually different selective histories for each arrangement.

A comparison of the various fitness estimates in the literature for these particular AR and $\mathrm{CH}$ chromosomes (table 2) is not very encouraging if the goal we are seeking is some universal measure of fitness. Such estimates are seen to differ between natural and laboratory populations, between the karyotypes of monomorphic and polymorphic laboratory populations, and between different techniques for determining fitness. Indeed, it would appear that we are in a position similar to the fable of the blind men and the elephant: each blind man produced a different description of the elephant depending on which part he grabbed on to. In the present case, there is the additional complication that the elephant is evidently in motion, i.e., for some populations, changes in fitness appear to be continually taking place (Strickberger, I963).

We may, of course, be unable to discern as yet a " good " empirical measure which would enable us to predict the relative fitness of each of these populations and genotypes under different conditions. Or, on the other hand, there may really be no such discernible value because of an " uncertainty principle" in the determination of fitness. That is, once we change the conditions of a population so that we can measure one of its characteristics, we may also change the relative fitness of the population. This idea is not unique (Lerner, 1955; Mayr, I955) and may be sufficient to account for the disparity between these data.

\section{SUMMARY}

The comparative productivity of experimental populations of Drosophila pseudoobscura was estimated in terms of the biomass of offspring produced weekly in half-pint culture bottles by approximately 
75 pairs of parents. Three different populations of third chromosome karyotypes, AR/CH, AR/AR, $\mathrm{CH} / \mathrm{CH}$, derived from a common polymorphic population cage, I 73, were tested in conjunction with a population derived from a cage monomorphic for the $\mathrm{CH}$ arrangement, I77. All the I73 populations were superior in productivity to the I77 population, thereby reversing the previously determined order of fitness under competitive conditions. Within the I 73 group, the $\mathrm{AR} / \mathrm{CH}$ heterokaryotype populations appeared superior to the homokaryotypes. There was no differential increase in population size when, towards the end of the experiment, AR chromosomes from one of the I 73 populations were periodically added to one of the I 73 $\mathrm{CH}$ populations. These findings are discussed from the view that "coadaptation" or mutual compatibility occurs between karyotypes of the same polymorphic population but can be significantly modified by individually different selective histories for these karyotypes over a period of time.

The absence of simple agreement between various estimates of fitness for the particular chromosomes used may be accounted for on the basis of an "uncertainty principle" which involves the occurrence of unpredictable changes in the relative fitness of a population during the conditions of measurement.

Acknowledgments.- The experiment was done at the Zoology Department of Columbia University while the author held a pre-doctoral fellowship with the U.S. Public Health Serrvice. The manuscript was written during a post-doctoral traineeship provided by the Public Health Service to the Genetics Department, University of California, Berkeley. I am indebted to Professors T. Dobzhansky and I. M. Lerner for their kind hospitality and sponsorship, and owe an additional debt to the latter for a critical reading of the manuscript. Mrs O. Pavlovsky and Dr Angela Solima Simmons very kindly maintained and scored the populations during a vacation period.

\section{REFERENCES}

BEARDMORE, J. A., DOBZhaNSKy, TH., AND PAVlovsky, o. ig6o. An attempt to compare the fitness of polymorphic and monomorphic experimental populations of Drosophila pseudoobscura. Heredity, 14, 19-33.

BUzzati-TRAVERSo, A. A. 1955. Evolutionary changes in components of fitness and other polygenic traits in Drosophila melanogaster populations. Heredity, 9, 153I 86.

CARsON, H. L. 1958. Increase in fitness in experimental populations resulting from heterosis. Proc. Nat. Acad. Sci., 44, I I 36-I I 4 I.

CARson, H. L. Ig6ra. Heterosis and fitness in experimental populations of Drosophila melanogaster. Evolution, 15, 496-509.

CARSON, H. L. I96I $b$. Relative fitness of genetically open and closed experimental populations of Drosophila robusta. Genetics, 46, 533-567.

DOBZHANSKY, TH. 1947. A directional change in the genetic constitution of a natural population of Drosophila pseudoobscura. Heredity, $I, 53-64$.

DoBZHANSKY, TH. I950. Genetics of natural populations. XIX. Origin of heterosis through natural selection in populations of Drosophila pseudoobscura. Genetics, $35,288-302$.

DOBZHANSKY, TH., AND PAVLovsKy, o. 196o. How stable is balanced polymorphism ? Proc. Nat. Acad. Sci., 46, 4 i-47. 
Dobzhansky, TH., aNd Pavlovsky, o. 1961. A further study of fitness of chromosomally polymorphic and monomorphic populations of Drosophila pseudoobscura. Heredity, $16,169-177$.

Gustafsson, A. 1951. Mutations environment and evolution. Cold Sp. Harb. Symp., $16,263-281$.

Lerner, I. M. 1955. Concluding survey. Cold. Sp. Harb. Symp., 20, 334-340.

LERNER, I. M., AND Ho, F. K. 1961. Genotype and competitive ability of Tribolium species. Amer. Nat., 95, 329-343.

mather, K. 1961. Competition and co-operation. Symp. Soc. Expt. Biol., 15, $264-281$.

MAYR, E. 1955. Integration of genotypes: synthesis. Cold. Sp. Harb. Symp., 20, 327-333.

montgomery, E. G. 1912. Competition in cereals. Bull. Neb. Agric: Exp. Sta., $127,3-22$.

SAKAI. K., AND GOTOH, K. 1955. Studies on competition in plants. IV. Competitive ability of $F_{1}$ hybrids in barley. Heredity, $46,139-143$.

STRICKBERGER, M. W. 1963. Evolution of fitness in experimental populations of Drosophila pseudoobscura. Evolution, $17,40-55$. 